\title{
Do patients with demyelinating diseases have antibodies against human glial cells in their sera?
}

\author{
P G E KENNEDY* ANDR P LISAK $\dagger$ \\ From the Medical Research Council Neuroimmunology Project, Department of Zoology, \\ University College, London
}

SUMMARY Cell-type-specific markers and indirect immunofluorescence were used to study immunoglobulin binding to glial cells in dissociated cell cultures of human foetal optic nerve, spinal cord and dorsal root ganglion in sera from patients with demyelinating diseases, other neurological diseases and normal controls. These various sera proved to be indistinguishable in that almost all of them contained immunoglobulin which bound weakly to all oligodendrocytes and fibroblasts, $5-25 \%$ of astrocytes and about $50 \%$ of Schwann cells.

The evidence for serum antibodies directed against myelin-producing cells in patients with multiple sclerosis (MS) is controversial. Whereas Abramsky et $a l^{1}$ demonstrated serum antibodies to oligodendrocytes that appeared to be specific to multiple sclerosis patients, Traugott et $a l^{2}$ and Kennedy and Lisak $^{3}$ were unable to distinguish between patients with demyelinating diseases and controls in terms of serum anti-glial cell antibodies. However, none of these studies used human glial cells in culture as target antigens. Clearly, an important question to answer is whether such antibodies can be demonstrated when cultured human glial cells are exposed to sera from patients with multiple sclerosis. For this purpose we developed techniques for growing and maintaining in culture human glial cells isolated from human foetal nervous tissues. ${ }^{4}$ We have shown that the same cell-type-specific markers previously used for identifying glial cells in cultures of neonatal rat nervous tissues ${ }^{5}$ can be used in cultures of human foetal central nervous system (CNS) tissues. ${ }^{4}$ For example, galactocerebroside (GC), the major glycolipid in myelin, ${ }^{7}$ has been shown to be expressed only by oligodendro-

* Supported by the National Fund for Research into Crippling Diseases. † Supported by US-UK Joint Education Commission (Fulbright-Hays).

Address for reprint requests: Dr PGE; Kennedy, Department of Zoology, University College London, Gower Street, London WC1E 6BT.

Accepted 22 October 1980 cytes in cultures of neonatal $\mathrm{rat}^{5}$ and human foetal" CNS tissues, while the "glial fibrillary acidic protein" (GFAP) has been shown to be expressed only by astrocytes in such cultures. ${ }^{45}$ We now report the findings of a search for antibodies directed against human glial cells in the sera of patients with neurological diseases.

\section{Patients and methods}

\section{Patients}

Serum specimens were obtained from a total of 37 subjects. Fourteen of the 15 patients with multiple sclerosis were clinically definite and one was early probable or latent. ${ }^{8}$ Six had a chronic progressive course, five were in acute exacerbation, and four were stable cases. Two of the patients in acute exacerbation were receiving corticosteroids. The other diseases studied included Guillain-Barré syndrome (seven cases), amyotrophic lateral sclerosis (three cases), chronic demyelinating peripheral neuropathy (one case) and systemic lupus erythematosis with CNS involvement (one case). All of the patients with GuillainBarré syndrome were in the acute stage of the disease and none were receiving corticosteroids. Sera were also obtained from 10 normal individuals.

\section{Cell cultures}

Dissociated primary cell cultures of human foetal spinal cord, optic nerve and dorsal root ganglion were prepared from 15-21-week-old human foetuses as previously described. ${ }^{4}$ In summary, whole optic nerves small segments of spinal cord or dorsal root ganglion were dissected from aborted foetuses as soon as poss- 
ible after death. The tissue was cut into small pieces, digested three times in trypsin and collagenase, dissociated by repeated trituration through either a Pasteur Pipette (optic nerve and spinal cord) or a no 23 hypodermic needle (dorsal root ganglion), and passed through sterile nylon gauze. The resultant turbid cell suspension was plated on $13 \mathrm{~mm}$ glass coverslips in Dulbecco's Modified Eagle's Medium containing $20 \%$ foetal calf serum and $50 \mu \mathrm{g}$ gentamicin (Flow Labs) in Linbro Multiwell plates (Flow Labs). Cells were studied after 2-5 days in culture.

\section{Indirect immunofluorescence}

Indirect immunofluorescence assays were performed as previously described. ${ }^{346}$ All of the antisera were heated at $56^{\circ} \mathrm{C}$ for 30 minutes before use. The cells growing on glass coverslips were incubated with patients' or normal serum (diluted $1: 10$ ) for $25 \mathrm{~min}$ utes at room temperature, washed well and then exposed to goat anti-human IgG conjugated to rhodamine ( $\mathrm{G}$ anti-HIg-Rd, Nordic, batch 10-374, diluted $1: 40$ ). After washing, the cells were fixed in $5 \%$ glacial acetic acid in $95 \%$ ethanol (acid-alcohol), washed and mounted in glycerol on glass slides. The cells were then examined under a Zeiss Universal fluorescence microscopic equipped with epiillumination, phase contrast and Nomarski interference optics. Staining of cells was graded 0 to ++++ .

Fifty per cent of sera were studied using twofluorochrome immunofluorescence as previously described. ${ }^{36}$ In these experiments cells were exposed to two antisera simultaneously, followed by the appropriate anti-human and anti-rabbit fluorochromeconjugated antibodies. Rabbit anti-GC and rabbit antiGFAP antibodies were used to identify oligodendrocytes and astrocytes respectively. For labelling with rabbit anti-GFAP, cells were first stained live with human serum, fixed in acid-alcohol, and then stained for GFAP. Schwann cells were identified by their characteristic bipolar morphology and by their expression of GC during the first three days in culture. ${ }^{4}$ Antibody binding was visualised by $\mathrm{G}$ anti-HIg-Rd, goat anti-human IgG conjugated to fluorescein (G anti-HIg-Fl, Nordic, batch 19-1075, diluted 1:40), and goat anti-rabbit IgG conjugated to rhodamine ( $\mathrm{G}$ anti-RIg-Rd, Nordic, batch 5-874, diluted 1:50) or fluorescein (G anti-RIg-Fl, Nordic, batch 3-277, diluted $1: 100)$.In double-labelling experiments $\mathrm{G}$ anti-HIgFl was adsorbed with rabbit immunoglobulin coupled to Sepharose. ${ }^{3}$
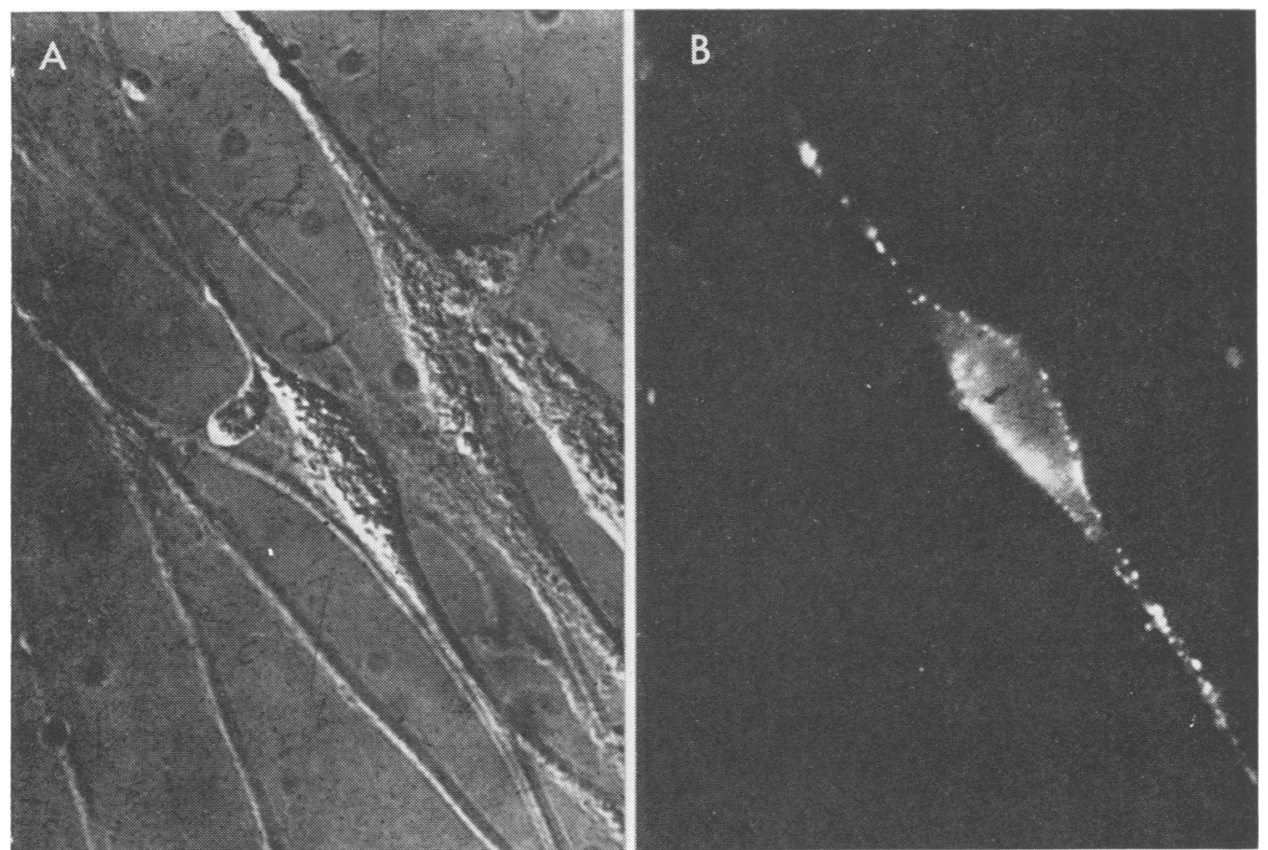

Figure Labelling of human foetal Schwann cell by multiple sclerosis serum. After 3 days cells from human dorsal root ganglian cultures were exposed to multiple sclerosis serum follewed by $G$ anti-HIg-Rd and viewed with Nomarski $(A)$ or rhodamine fluorescence $(B)$ optics. One of the bipolar Schwann cells is labelled. 


\section{Results}

\section{(i) Spinal cord and optic nerve cultures}

The spinal cord and optic nerve cultures used in this study contained approximately $25 \%$ GFAP+ astrocytes, 4\% GC+ + oligodendrocytes and 70\% GFAP- fibroblastic cells. ${ }^{4}$ Fourteen of the multiple sclerosis sera tested in these cultures showed weak staining ( + or ++ ) of all oligodendrocytes and fibroblasts, and $5-25 \%$ of astrocytes. The other serum from patients with multiple sclerosis showed no staining of oligodendrocytes or astrocytes but weak staining of fibroblasts. All of the sera from patients with other neurological diseases and normal controls showed a similar pattern of weak staining of oligodendrocytes, astrocytes and fibroblasts. One of the sera, from patients with Guillain-Barré syndrome, however, showed particularly bright staining $(++++)$ of fibroblasts.

When higher dilutions $(1: 40$ and $1: 100)$ of representative patients' sera were tested there was still no difference between patients with multiple sclerosis and controls, although at $1: 100$ dilution the staining of individual cells was very weak. Fixing the cells in acid-alcohol prior to staining with representative sera produced cytoplasmic staining of glial cells and fibroblasts which was similar with sera from patients with multiple sclerosis.

\section{(ii) Dorsal root ganglion cultures}

Dorsal root ganglion cultures contained approximately $30 \%$ bipolar Schwann cells, $10 \%$ Tetanus toxin $^{+}$neurones, and $50 \%$ GFAP $^{-}$fibroblastic cells. ${ }^{4}$ More than $80 \%$ of the GFAP ${ }^{+}$fibroblastic cells were Thy-l-like antigen +.4

Two (one patient with Guillain-Barré syndrome and one normal) of the 18 representative sera tested in these cultures showed no staining of Schwann cells but weak staining $(+)$ of fibroblasts. All of the other sera tested were indistinguishable in their pattern of binding: they all showed weak staining $(+$ or ++ ) of about $50 \%$ of Schwann cells (figure) and all fibroblasts. This type of staining was seen in both two- and five-day-old dorsal root ganglion cultures.

\section{Discussion}

In this study we have employed cell-type-specific markers and indirect immunofluorescence to show that the sera of patients with demyelinating diseases, other neurological diseases and normal individuals are indistinguishable in that they all bind to cultured human foetal glial cells. Virtually all of the sera tested showed weak surface immunoglobulin binding to oligodendrocytes, fibroblasts and some astrocytes in spinal cord and optic nerve cultures, and most of the sera also reacted weakly with fibroblasts and about $50 \%$ of Schwann cells in dorsal root ganglion cultures. These findings are similar to our previous studies ${ }^{3}$ in which the same sera were tested in short-term non-myelinated cultures of neonatal and adult rat central and/or peripheral nervous tissues. In the latter studies we observed the same pattern of binding of all sera to oligodendrocytes, fibroblasts, astrocytes and Schwann cells, although a smaller proportion of astrocytes were labelled compared to the present study. In addition, the staining of fibroblasts was, in general, more intense in rat cultures.

Traugott et $a l^{2}$ have recently reported similar findings using indirect immunofluorescence on frozen sections of human and bovine brain and isolated bovine oligodendrocytes in suspension. They found that oligodendrocyte staining in these tissues was not specific to MS as it was also detected in a large number of sera from patients with other neurological diseases and normal controls. Immunofluorescent staining of astrocytes was also shown by all groups. While the reasons for the differences between our results and those of Abramsky et $a^{1}{ }^{1}$ are still unclear, ${ }^{3}$ the present study lends further support to the notion that autoantibodies to myelin-producing cells are unlikely to be of importance in the pathogenesis of multiple sclerosis or Guillain-Barré syndrome.

We would like to thank Professor MC Raff for his advice and helpful discussions, and Drs $S$ Cotmore and RM Pruss for their kind gifts of antisera. Permission to work with human foetal material was granted by the Ethical Committees of University College, London and University College Hospital.

\section{References}

1 Abramsky O, Lisak RP, Silberberg DH, Pleasure DE. Antibodies to oligodendroglia in patients with multiple sclerosis. New Engl J Med 1977; 297:1207-11.

2 Traugott U, Snyder DS, Raine CS. Oligodendrocyte staining by multiple sclerosis serum is nonspecific. Ann Neurol 1979; 6:13-20.

3 Kennedy PGE, Lisak RP. A search for antibodies against glial cells in the serum and cerebrospinal fluid of patients with multiple sclerosis and Guillain-Barré syndrome. J Neurol Sci 1979; 44: 125-33. 
4 Kennedy PGE, Lisak RP, Raff MC. Cell-typespecific markers for human glial and neuronal cells in culture. Lab Invest 1980; 43:342-51.

5 Raff MC, Mirsky R, Fields et al. Galactocerebroside is a specific cell surface antigenic marker for oligodendrocytes in culture. Nature 1978; 274:813-6.

6 Raff MC, Fields KL, Hakomori S, Mirsky R, Pruss, RM, Winter J. Cell-type-specific markers for distinguishing and studying neurons and the major classes of glial cells in culture. Brain Res 1979; 174:283-308.

7 Norton WT, Autilio LA. The lipid composition of purified bovine brain myelin. J Neurochem 1966; 13:213-22.

8 McDonald WI, Halliday AM. Diagnosis and classification of multiple sclerosis. Brit Med Bull 1977; 33:4-9. 